\title{
Enhanced Sampling Applied to Modeling Allosteric Regulation in Transcription
}

Yanming Wang ${ }^{1}$ and Charles L. Brooks III ${ }^{1,2 *}$

1. Department of Chemistry and 2. Biophysics Program, University of Michigan, Ann Arbor, Michigan 48109, United States

\section{Corresponding Author}

*Correspondence to: brookscl@umich.edu 


\section{Supporting Information}

\section{SI Text}

The EIKB Model. The structure-based EIKB models of the two ternary systems c-Myb:KIX:MLL and pKID:KIX:MLL were built from the first frame of PDB files 2AGH and 2LXT using the protocol described previously. ${ }^{1}$ The KB model, i.e., that without included electrostatic interactions, was originally designed to study protein folding and uses a single bead to represent each amino acid residue. ${ }^{2}$ The force field includes bonded and non-bonded interactions. The bonded interactions include bond, angle and dihedral terms. The bond and angle terms are biased to the native structure while the dihedral term is "sequence-flavored" and is not biased to native conformation to reduce potential overfitting. The non-bonded interactions consider the native contacts and the excluded volume interactions. Native contacts are defined as those formed between two residues of which the closest non-hydrogen atoms are within $4.5 \AA$ or two residues that form hydrogen bond(s). The native contact interactions are modeled using a modified Lennard-Jones potential with desolvation penalty ${ }^{1}$. The Miyazawa-Jernigan $(\mathrm{M}-\mathrm{J})^{3}$ contact strengths were first used as the baseline of the native contact strengths. The strengths of all native contact interactions were then scaled to keep the folding temperature around $350 \mathrm{~K}^{1}$.

The original KB Gō model did not consider electrostatics since the M-J contact interactions, which represent a statistical potential, already include some electrostatic effects. The electrostatic effect is also relatively less important than the hydrophobic effect, which is represented by native contacts in the Gō model, for protein folding. However, IDPs are often rich in charged residues and our data suggested that electrostatics is essential to capture the positive allostery in the pKID:KIX:MLL system. The electrostatics inclusive KB model (EIKB model) uses a simple Debye-Hückel potential (equation 1) to mimic the long-range electrostatics. This type of potential 
was shown to be compatible with the coarse-grained model. ${ }^{4,5}$ A screening length $\zeta=10 \AA$ was chosen to mimic the salt screening effect. The dielectric constant D was tested at 40 and 80 to represent strong and weak electrostatic environments. Our study suggests that $\mathrm{D}=40$ is important for the positive allosteric effect in pKID:KIX:MLL. The model only considers the electrostatic interactions among charged residues: ASP (-1), GLU (-1), HIS (+0.5), LYS (+1), and ARG (+1). For pKID, the phosphoserine SER133 was modeled as glutamic acid similar to ref. 6. To avoid double-counting, salt-bridge native contact interactions were scaled down by $40 \%$ similar to previous work. ${ }^{6}$ The previous study published by our group ${ }^{7}$ on c-Myb:KIX:MLL used the KB model without electrostatics but also captured the positive allosteric effect. The success of the KB model without electrostatics on c-Myb:KIX:MLL is due to the weak electrostatic interactions between c-Myb and MLL in native conformation (Table S2). Therefore, electrostatics is unimportant for the positive allostery in c-Myb:KIX:MLL (Figures 3 and S1).

$$
V_{\text {elec }}=332 \frac{q_{1} q_{2}}{D r} \exp \left(-\frac{r}{\zeta}\right)
$$

The original KB model uses the enthalpy-entropy compensation paradigm to scale all native contacts to keep the folding temperature around $350 \mathrm{~K} .{ }^{1}$ The native contact strengths were scaled so that the sum of all native contact energies in the native conformation is equal to the free energy contribution of the empirically calculated entropy loss from the unfolded state to the folded state. Therefore, for the IDP-protein system, the KB model overestimates the intramolecular interactions of IDPs while it underestimates the intermolecular interactions between IDPs and proteins. To address these issues, we adopted a calibration protocol from earlier studies. ${ }^{6,7}$ The native contacts were classified into the intramolecular $\left(\mathrm{V}_{\text {intra }}\right)$ and intermolecular $\left(\mathrm{V}_{\text {inter }}\right)$ contacts, with two scaling factors $\alpha$ and $\beta$ added on them, respectively (equation 2). The values of the scaling factor $\alpha$ were 
adopted from previous studies with $\alpha_{\mathrm{c}-\mathrm{Myb}}=0.45^{7}, \alpha_{\mathrm{MLL}}=0.05^{7}, \alpha_{\mathrm{pKID}-\alpha 1}=0.75^{6}$, and $\alpha_{\mathrm{pKID}-\alpha 2}=$ $0.15^{6}$. Those values were obtained by fitting the model with helicities from either experiment or bioinformatics predictions. The scaling factor $\beta$ for each IDP-protein system was determined using the HREX method described in the main text.

$$
V_{\text {native }}=\alpha V_{\text {intra }}+\beta V_{\text {inter }}
$$

Model Setup for c-Myb:KIX:MLL and pKID:KIX:MLL Systems. A total of four EIKB models of binary complexes (KIX $: \mathrm{c}-\mathrm{Myb}, \mathrm{KIX}_{\mathrm{c}}: \mathrm{MLL}_{\mathrm{c}}, \mathrm{KIX}_{\mathrm{p}}$ :pKID, and $\mathrm{KIX}_{\mathrm{p}}: \mathrm{MLL}_{\mathrm{p}}$ ) and two models of ternary complexes were built and simulated in this study. The subscript "c" and "p" denote structures built from c-Myb:KIX:MLL (PDB code: 2AGH) and pKID:KIX:MLL (PDB code 2LXT), respectively. First, the force fields of ternary complexes were built from the PDB structures. Then, the force fields of binary complexes were directly extracted from their ternary models. One assumption of building the binary model based on the ternary model is that the structure of the binary complex is identical to that in the corresponding ternary complex. This assumption is supported by NMR studies ${ }^{8}$ in which the pairwise RMSD between backbone atoms of the well-structured parts of the KIX domain in the KIX MLL $_{p}$ binary complex and pKID:KIX $:$ MLL $_{\mathrm{p}}$ ternary complex is only $1.07 \AA$ and the KIX protein backbone is not significantly affected by binding pKID. Ideally, the EIKB models of KIX $: \mathrm{MLL}_{\mathrm{c}}$ (built from cMyb:KIX:MLL) and KIX $:$ MLL $_{p}$ (built from pKID:KIX:MLL) should be the same. However, they are treated differently due to the native-centric nature of the EIKB model. The native contacts used by the EIKB models of $\mathrm{KIX}_{\mathrm{c}}: \mathrm{MLL}_{\mathrm{c}}$ and $\mathrm{KIX}_{\mathrm{p}}: \mathrm{MLL}_{\mathrm{p}}$ are listed in tables $\mathrm{S} 4$ and $\mathrm{S} 5$, respectively. A total of 10 native contacts are found in common (marked as red) out of the 28 intermolecular native contacts of $\mathrm{KIX}_{\mathrm{c}}: \mathrm{MLL}_{\mathrm{c}}$ and the 27 intermolecular native contacts of $\mathrm{KIX}_{\mathrm{p}}: \mathrm{MLL}_{\mathrm{p}}$. The RMSD between the EIKB models of $\mathrm{KIX}_{\mathrm{c}}: \mathrm{MLL}_{\mathrm{c}}$ and $\mathrm{KIX}_{\mathrm{p}}: \mathrm{MLL}_{\mathrm{p}}$ is $3.72 \AA$. Given the facts that 
$\mathrm{KIX}_{\mathrm{c}}: \mathrm{MLL}_{\mathrm{c}}$ and $\mathrm{KIX}_{\mathrm{p}}: \mathrm{MLL}_{\mathrm{p}}$ are structurally similar, their EIKB models show reasonable number of identical native contacts $(>30 \%)$, and the sums of force constants of the intermolecular native contacts for both $\mathrm{KIX}_{\mathrm{c}}: \mathrm{MLL}_{\mathrm{c}}\left(\mathrm{sum}=-25.50 \mathrm{kcal} / \mathrm{mol} / \AA^{2}\right)$ and $\mathrm{KIX}_{\mathrm{p}}: \mathrm{MLL}_{\mathrm{p}}(\mathrm{sum}=-27.24$ $\mathrm{kcal} / \mathrm{mol} / \AA^{2}$ ) are very close, it is reasonable to assume the EIKB models of $\mathrm{KIX}_{\mathrm{c}}: \mathrm{MLL}_{\mathrm{c}}$ and $\mathrm{KIX}_{\mathrm{p}}: \mathrm{MLL}_{\mathrm{p}}$ are approximately the same. In fact, our simulations reproduced the positive allosteric effects in both c-Myb:KIX:MLL and pKID:KIX:MLL. Therefore, it is reasonable to assume that this approximation does not affect the validity of our conclusions.

The HREX Implementation and Molecular Dynamics Protocol. All simulations were performed using the OpenMM library ${ }^{10}$, which allows simulations with highly customizable force fields and GPU acceleration. The HREX method shows some resemblance with the Replica Exchange with Solute Tempering (REST) method $^{9}$ in which scaling factors are used to control interactions between different groups of atoms. The HREX method was implemented using inhouse $\mathrm{C}++$ codes based on the OpenMM C++ API and Message Passing Interface (MPI). Each MPI process runs a single simulation. Two Hamiltonian variables, temperature $\mathrm{T}$ and the scaling factor $\beta$, were chosen as exchange coordinates. The HREX method allows replicas to exchange coordinates with each other and therefore enhances the sampling. The system cartesian coordinates of the two nearest windows $\left(T_{i}, T_{i+1}\right)$ or $\left(\beta_{i}, \beta_{i+1}\right)$ were exchanged every 10,000 steps using the Metropolis algorithm. ${ }^{11}$ The average exchange rate (number of successful exchanges / total number of exchange trials) is $24.86 \%$ for all HREX simulations in Figure 2. Each HREX simulation uses $11 \beta$ windows covering a range of systems from low binding affinity to high binding affinity. The $\beta$ windows are shown in Figures 2 and S1. Three temperature windows at $300 \mathrm{~K}, 320 \mathrm{~K}$, and 340 $\mathrm{K}$ were used for each HREX simulation. Therefore, a total of 33 replicas were used for a single HREX simulation. Only the replicas at $300 \mathrm{~K}$ were used for data analysis. Use of MBAR or 
WHAM could further enhance the precision of our calculations by including the other temperature; however, because we were able to achieve sufficient precision without adding these other temperatures in, we did not include them here. During the simulation, all bonds were kept constrained to their native distances. The force field of the EIKB model was implemented using different force objects of the OpenMM library and is already described in the Model section. The Langevin integrator was used to propagate the equations of motion with a friction coefficient of $0.1 \mathrm{ps}^{-1}$. For each HREX simulation, 400 million steps were simulated for each replica with a time step of 22 fs. The first 50 million steps were discarded in the data analysis. For the unbiased simulation, 3 billion steps were simulated for each system with the $\beta^{\text {opt }}$ obtained from the HREX simulation while other parameters were the same as HREX. For all trajectories, snapshots were collected every 10,000 steps for data analysis. Periodic boundary condition with a cubic box of $150 \AA$ was used for all simulations. All HREX simulations were repeated 3 times, and the unbiased simulations were repeated 10 times for each system of interest.

Trajectory Analysis. The fraction of native contacts, Q, is computed as the fraction of native contact pairs of which the distance is within $\left(R_{i j}+1\right) \AA$, where $R_{i j}$ is the native contact distance between residue $\mathrm{i}$ and residue $\mathrm{j}$ in the native conformation. The fraction of unbound states $\mathrm{P}_{\mathrm{u}}$ of each trajectory was calculated as the fraction of snapshots with $\mathrm{Q}<0.1$. The dissociation constant $\mathrm{K}_{\mathrm{d}}$ is calculated using equation (3), where $\mathrm{V}$ is the volume of the periodic box.

$$
K_{d}=\frac{1660}{V} \times \frac{P_{u}^{2}}{1-P_{u}}
$$

Data Analysis. The relationship between the dissociation constant $K_{d}$ and the scaling factor $\beta$ that tunes the intermolecular interaction strengths is shown by equation (4). The derivation of equation (4) is already shown in the main text. In this equation, $\Delta \mathrm{S}$ is the total entropy change, and $\Delta \mathrm{H}$ is 
the total enthalpy change. The total entropy or enthalpy may be decomposed into the entropy/enthalpy from binding and other contributions: $\Delta \mathrm{S}=\Delta \mathrm{S}_{\text {bind }}+\Delta \mathrm{S}_{\text {other }}$ and $\Delta \mathrm{H}=\Delta \mathrm{H}_{\text {bind }}+$ $\Delta \mathrm{H}_{\mathrm{other}}$. One assumption of equation (4) is that $\Delta \mathrm{S}_{\mathrm{other}} / \Delta \mathrm{H}_{\mathrm{other}}$ does not change before and after binding. This is a reasonable assumption since our models contain only configurational degrees of freedom, and equation (4) fits the data very well. For clarity, $\Delta S / \Delta H$ is used as the binding entropy/enthalpy with a constant offset added, which does not affect our data analysis. The nonlinear fitting was performed using the "scipy.optimize.curve_fit()" function in the Python SciPy package. The calculation of the HREX-based conformational entropy/enthalpy was described in the main text. The calculation of the order parameter (OP) based conformational entropy was described in earlier work. ${ }^{7}$ The OP-based conformational entropy was calculated using the 3D histogram of three order parameters from the unbiased simulations with $\beta^{\text {opt }}$ obtained from HREX calculations. All thermodynamic parameters were calculated at $300 \mathrm{~K}$. Error bars are estimated as $+/-$ the standard deviation (std) of the data, where $\operatorname{std}=\operatorname{sqrt}(\operatorname{sum}((\mathrm{x}-\operatorname{mean}(\mathrm{x})) * * 2) / \operatorname{len}(\mathrm{x})$ and $\mathrm{x}$ is the data array using the Python syntax.

$$
\ln \left(K_{d}\right)=\ln \left(\frac{1660}{V}\right)-\frac{T \Delta S}{R T}+\frac{\Delta H}{R T} \beta-\ln \left\{\exp \left(\frac{T \Delta S}{R T}-\frac{\Delta H}{R T} \beta\right)+1\right\}
$$




\section{SI Figures}
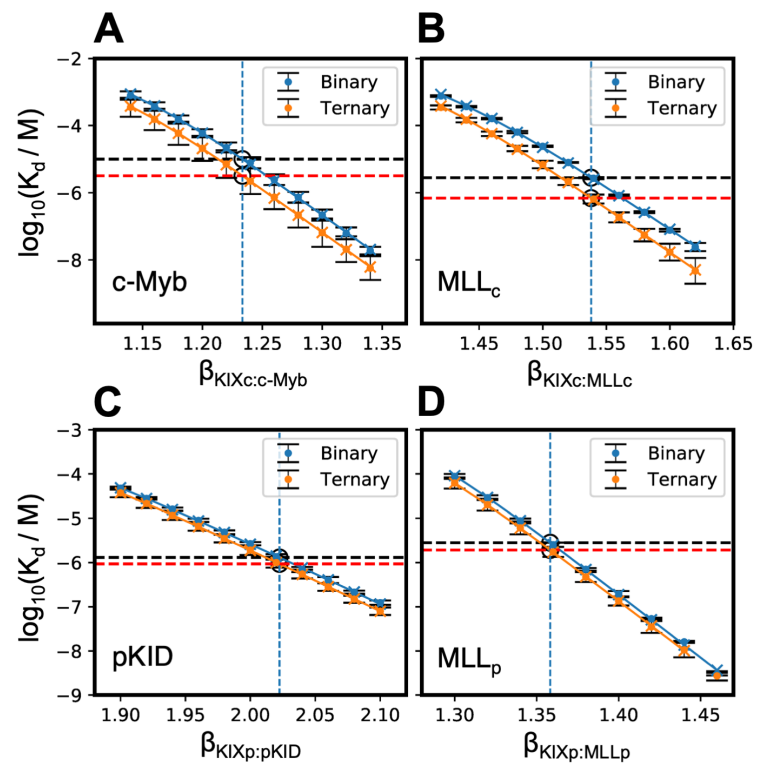

Figure S1. Calibration curves of the binary and ternary systems at $\mathrm{D}=80$. Each panel represents IDP calibration curves in the binary or ternary complexes.

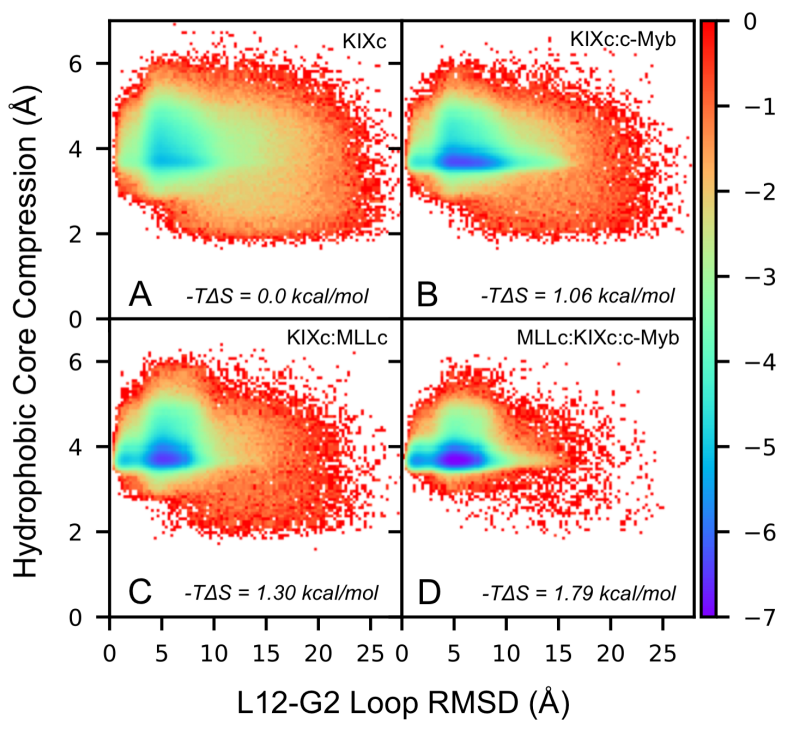

Figure S2. Free energy plots of the hydrophobic core compression and $\mathrm{L}_{12}-\mathrm{G}_{2}$ loop RMSD of KIX in the free state (A), bound with c-Myb (B), bound with $\mathrm{MLL}_{c}(\mathrm{C})$, and bound with both c-Myb and $\mathrm{MLL}_{\mathrm{c}}$ (D). Definitions of these order parameters are described in ref 7. 


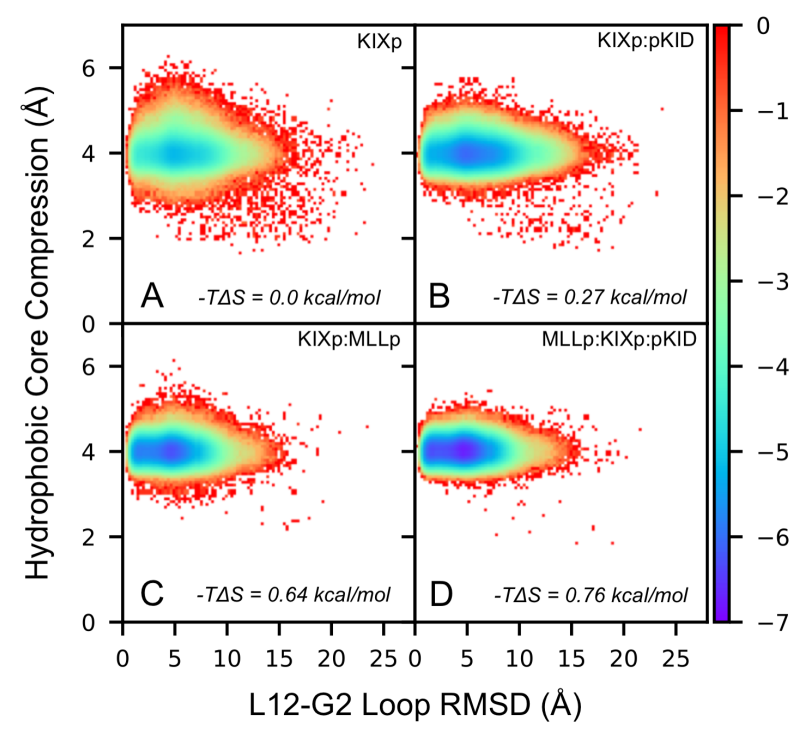

Figure S3. Free energy plots of the hydrophobic core compression and $\mathrm{L}_{12}-\mathrm{G}_{2}$ loop RMSD of KIX in the free state (A), bound with pKID (B), bound with $\mathrm{MLL}_{c}(\mathrm{C})$, and bound with both c-Myb and $\mathrm{MLL}_{\mathrm{c}}(\mathrm{D})$. Definitions of these order parameters are described in ref 7. 


\section{SI Tables}

Table S1. $K_{d}$ S of different systems calculated by HREX and unbiased simulations at D = 40 .

\begin{tabular}{ccccc}
\hline Ligand & Binding to & Exp. $\mathrm{K}_{\mathrm{d}}, \mu \mathrm{M}^{\mathrm{a}}$ & $\mathrm{HREX} \mathrm{K}_{\mathrm{d}}, \mu \mathrm{M}^{\mathrm{b}}$ & Unbiased $_{\mathrm{d}}, \mu \mathrm{M}^{\mathrm{c}}$ \\
\hline $\mathrm{c}-\mathrm{Myb}$ & $\mathrm{KIX}_{\mathrm{c}}$ & $10 \pm 2$ & 10 & $27.7 \pm 26.1$ \\
$\mathrm{c}-\mathrm{Myb}$ & $\mathrm{KIX}_{\mathrm{c}}: \mathrm{MLL}_{\mathrm{c}}$ & $4 \pm 1$ & 2.19 & $7.28 \pm 12.7$ \\
MLL $_{\mathrm{c}}$ & $\mathrm{KIX}_{\mathrm{c}}$ & $2.8 \pm 0.4$ & 2.8 & $3.69 \pm 1.22$ \\
MLL $_{\mathrm{c}}$ & $\mathrm{KIX}_{\mathrm{c}}: \mathrm{MLL}_{\mathrm{c}}$ & $1.7 \pm 0.1$ & 0.56 & $0.76 \pm 0.36$ \\
$\mathrm{pKID}$ & $\mathrm{KIX}_{\mathrm{p}}$ & $1.30 \pm 0.02$ & 1.30 & $1.73 \pm 0.98$ \\
pKID & $\mathrm{KIX}_{\mathrm{p}}: \mathrm{MLL}_{\mathrm{p}}$ & $0.65 \pm 0.05$ & 0.63 & $0.66 \pm 0.52$ \\
MLL $_{\mathrm{p}}$ & $\mathrm{KIX}_{\mathrm{p}}$ & $2.8 \pm 0.4$ & 2.8 & $4.08 \pm 1.46$ \\
MLL $_{\mathrm{p}}$ & $\mathrm{KIX}_{\mathrm{p}}: \mathrm{pKID}$ & $1.5 \pm 0.2$ & 1.08 & $1.15 \pm 0.69$
\end{tabular}

a. Experimental data. ${ }^{12}$

${ }^{b} . \mathrm{K}_{\mathrm{d}}$ calculated by HREX.

c. $\mathrm{K}_{\mathrm{d}}$ calculated by long unbiased simulations. 
Table S2. Electrostatic interactions between c-Myb and MLL in PDB structure 2AGH at D $=40$.

\begin{tabular}{|c|c|c|c|}
\hline Residues of c-Myb & Residues of MLL & Distance $(\AA)$ & Energy $(\mathrm{kcal} / \mathrm{mol})^{\mathrm{a}}$ \\
\hline LYS291 & ASP841 & 22.83 & -0.0371 \\
\hline LYS296 & ASP841 & 23.82 & -0.0322 \\
\hline ARG294 & ASP841 & 24.27 & -0.0302 \\
\hline ARG294 & ASP851 & 24.87 & -0.0277 \\
\hline LYS291 & ASP840 & 24.89 & -0.0277 \\
\hline LYS296 & ASP840 & 25.10 & -0.0269 \\
\hline GLU297 & LYS855 & 25.51 & -0.0254 \\
\hline GLU299 & LYS855 & 25.75 & -0.0246 \\
\hline LYS291 & ASP851 & 26.07 & -0.0235 \\
\hline ARG294 & ASP840 & 26.32 & -0.0227 \\
\hline LYS293 & ASP841 & 26.46 & -0.0223 \\
\hline GLU292 & LYS855 & 26.74 & -0.0214 \\
\hline ARG294 & ASP848 & 27.68 & -0.0188 \\
\hline LYS296 & ASP851 & 27.82 & -0.0185 \\
\hline LYS293 & ASP840 & 28.20 & -0.0175 \\
\hline LYS293 & ASP851 & 28.56 & -0.0167 \\
\hline LYS291 & ASP848 & 29.46 & -0.0148 \\
\hline LYS296 & ASP848 & 29.76 & -0.0142 \\
\hline LYS293 & ASP848 & 31.41 & -0.0114 \\
\hline GLU306 & LYS855 & 33.49 & -0.0087 \\
\hline GLU308 & LYS855 & 33.77 & -0.0084 \\
\hline LYS310 & ASP848 & 36.33 & -0.0060 \\
\hline LYS310 & ASP841 & 36.47 & -0.0059 \\
\hline LYS310 & ASP840 & 36.75 & -0.0057 \\
\hline LYS310 & ASP851 & 37.27 & -0.0054 \\
\hline LYS310 & LYS855 & 38.09 & 0.0048 \\
\hline GLU308 & ASP840 & 35.13 & 0.0070 \\
\hline GLU308 & ASP841 & 34.31 & 0.0078 \\
\hline GLU308 & ASP851 & 33.73 & 0.0084 \\
\hline GLU308 & ASP848 & 33.63 & 0.0086 \\
\hline GLU306 & ASP851 & 32.52 & 0.0099 \\
\hline GLU306 & ASP848 & 31.80 & 0.0109 \\
\hline GLU306 & ASP840 & 31.25 & 0.0117 \\
\hline GLU292 & ASP848 & 30.98 & 0.0121 \\
\hline GLU306 & ASP841 & 30.86 & 0.0123 \\
\hline GLU297 & ASP848 & 28.52 & 0.0168 \\
\hline GLU292 & ASP851 & 28.09 & 0.0178 \\
\hline
\end{tabular}




\begin{tabular}{cccc}
\hline LYS296 & LYS855 & 27.19 & 0.0201 \\
GLU297 & ASP840 & 26.92 & 0.0209 \\
LYS293 & LYS855 & 26.82 & 0.0212 \\
GLU299 & ASP848 & 26.58 & 0.0219 \\
GLU297 & ASP851 & 26.53 & 0.0220 \\
GLU292 & ASP840 & 25.55 & 0.0252 \\
GLU299 & ASP851 & 25.47 & 0.0255 \\
GLU297 & ASP841 & 25.31 & 0.0261 \\
LYS291 & LYS855 & 24.38 & 0.0297 \\
GLU292 & ASP841 & 23.91 & 0.0318 \\
ARG294 & LYS855 & 23.24 & 0.0350 \\
GLU299 & ASP840 & 22.56 & 0.0386 \\
GLU299 & ASP841 & 21.43 & 0.0454 \\
\hline Total & & & 0.0178 \\
\hline
\end{tabular}

a. Electrostatic energy at the pair distance calculated from the native structure. Attractive interactions are negative. 
Table S3. Electrostatic interactions between pKID and MLL in PDB structure 2LXT at D $=40$.

\begin{tabular}{|c|c|c|c|}
\hline Residues of c-Myb & Residues of MLL & Distance $(\AA)$ & Energy $(\mathrm{kcal} / \mathrm{mol})^{\mathrm{a}}$ \\
\hline ASP840 & ARG124 & 10.26 & -0.290 \\
\hline ASP840 & ARG125 & 11.27 & -0.239 \\
\hline ASP840 & LYS123 & 12.35 & -0.195 \\
\hline ASP840 & ARG131 & 18.09 & -0.075 \\
\hline ASP840 & ARG135 & 19.22 & -0.063 \\
\hline ASP840 & ARG130 & 19.31 & -0.062 \\
\hline ASP840 & LYS136 & 21.87 & -0.043 \\
\hline LYS855 & GLU133 & 24.50 & -0.029 \\
\hline ASP848 & ARG124 & 25.57 & -0.025 \\
\hline ASP851 & ARG124 & 25.78 & -0.024 \\
\hline ASP851 & ARG131 & 26.22 & -0.023 \\
\hline ASP851 & LYS136 & 27.27 & -0.020 \\
\hline ASP851 & ARG135 & 27.62 & -0.019 \\
\hline ASP848 & ARG131 & 27.71 & -0.019 \\
\hline ASP848 & ARG125 & 28.03 & -0.018 \\
\hline ASP851 & ARG125 & 28.27 & -0.017 \\
\hline ASP848 & LYS123 & 28.43 & -0.017 \\
\hline ASP851 & LYS123 & 28.79 & -0.016 \\
\hline ASP848 & ARG135 & 28.97 & -0.016 \\
\hline LYS855 & ASP140 & 28.99 & -0.016 \\
\hline ASP848 & LYS136 & 29.08 & -0.016 \\
\hline ASP851 & ARG130 & 29.60 & -0.015 \\
\hline ASP848 & ARG130 & 30.90 & -0.012 \\
\hline LYS855 & ASP120 & 31.19 & -0.012 \\
\hline LYS855 & ASP144 & 31.91 & -0.011 \\
\hline LYS855 & GLU126 & 33.37 & -0.009 \\
\hline LYS855 & ASP116 & 33.81 & -0.008 \\
\hline LYS855 & LYS123 & 32.82 & 0.010 \\
\hline ASP848 & ASP144 & 32.53 & 0.010 \\
\hline LYS855 & ARG125 & 31.82 & 0.011 \\
\hline ASP851 & ASP144 & 31.80 & 0.011 \\
\hline LYS855 & ARG130 & 31.48 & 0.011 \\
\hline ASP848 & ASP140 & 30.43 & 0.013 \\
\hline ASP848 & GLU126 & 30.37 & 0.013 \\
\hline ASP851 & GLU126 & 30.16 & 0.013 \\
\hline
\end{tabular}




\begin{tabular}{cccc}
\hline LYS855 & ARG124 & 29.61 & 0.015 \\
ASP851 & ASP116 & 29.07 & 0.016 \\
ASP851 & ASP140 & 28.97 & 0.016 \\
LYS855 & ARG135 & 28.78 & 0.016 \\
ASP848 & ASP116 & 28.23 & 0.017 \\
LYS855 & ARG131 & 27.87 & 0.018 \\
LYS855 & LYS136 & 27.61 & 0.019 \\
ASP851 & ASP120 & 26.43 & 0.022 \\
ASP848 & GLU133 & 25.99 & 0.024 \\
ASP840 & ASP144 & 25.78 & 0.024 \\
ASP848 & ASP120 & 25.34 & 0.026 \\
ASP851 & GLU133 & 23.92 & 0.032 \\
ASP840 & ASP140 & 23.63 & 0.033 \\
ASP840 & GLU133 & 20.17 & 0.055 \\
ASP840 & GLU126 & 14.78 & 0.128 \\
ASP840 & ASP116 & 14.27 & 0.140 \\
ASP840 & ASP120 & 8.93 & 0.380 \\
\hline Total & & & -0.236 \\
\hline
\end{tabular}

a. Electrostatic energy with the distance calculated from the native structure. Attractive interactions are negative. 
Table S4. Native contacts interactions between $\mathrm{KIX}_{\mathrm{c}}$ and $\mathrm{MLL}_{\mathrm{c}}$ in PDB structure $2 \mathrm{AGH}$. Contacts that are the same as those found in $\mathrm{KIX}_{\mathrm{p}}: \mathrm{MLL}_{\mathrm{p}}$ are marked as red.

\begin{tabular}{|c|c|c|c|}
\hline Residues of KIX & Residues of MLL & Force constant $\left(\mathrm{kcal} / \mathrm{mol} / \AA^{2}\right)$ & Distance $(\AA)$ \\
\hline PHE612 & PHE852 & -1.444 & 9.15 \\
\hline PHE612 & VAL853 & -1.251 & 6.39 \\
\hline PHE612 & ASN856 & -0.746 & 8.82 \\
\hline PHE612 & THR857 & -0.851 & 8.20 \\
\hline PRO615 & ASN856 & -0.304 & 6.95 \\
\hline ARG624 & ASP848 & -0.456 & 10.14 \\
\hline ARG624 & ASP851 & -0.456 & 10.89 \\
\hline ARG624 & PHE852 & -0.792 & 7.74 \\
\hline MET625 & PHE852 & -1.305 & 8.48 \\
\hline ASN627 & ASP848 & -0.334 & 8.11 \\
\hline ASN627 & ILE849 & -0.644 & 6.39 \\
\hline LEU628 & ILE849 & -1.400 & 5.89 \\
\hline LEU628 & PHE852 & -1.448 & 8.23 \\
\hline LEU628 & VAL853 & -1.289 & 8.57 \\
\hline TYR631 & ILE844 & -1.044 & 10.08 \\
\hline TYR631 & LEU845 & -1.128 & 8.53 \\
\hline TYR631 & PRO846 & -0.635 & 8.43 \\
\hline TYR631 & ILE849 & -1.044 & 7.47 \\
\hline MET639 & ILE844 & -1.197 & 11.51 \\
\hline LYS656 & ILE844 & -0.599 & 7.53 \\
\hline LYS659 & ILE844 & -0.599 & 8.76 \\
\hline ILE660 & ILE844 & -1.301 & 8.06 \\
\hline LEU664 & LEU845 & -1.466 & 11.32 \\
\hline LEU664 & VAL853 & -1.289 & 8.70 \\
\hline LEU664 & THR857 & -0.863 & 7.66 \\
\hline ARG668 & LEU854 & -0.802 & 7.83 \\
\hline ARG668 & THR857 & -0.378 & 6.43 \\
\hline ARG668 & PRO858 & -0.338 & 5.10 \\
\hline Total & & -25.40 & \\
\hline
\end{tabular}


Table S5. Native contacts interactions between $\mathrm{KIX}_{\mathrm{p}}$ and $\mathrm{MLL}_{\mathrm{p}}$ in PDB structure 2LXT. Contacts that are the same as those found in $\mathrm{KIX}_{c}: \mathrm{MLL}_{c}$ are marked as red.

\begin{tabular}{|c|c|c|c|}
\hline Residues of $\mathrm{KIX}_{\mathrm{p}}$ & Residues of $\mathrm{MLL}_{\mathrm{p}}$ & Force constant $\left(\mathrm{kcal} / \mathrm{mol} / \AA^{2}\right)$ & Distance $(\AA)$ \\
\hline ILE611 & LEU845 & -1.610 & 10.45 \\
\hline ILE611 & MET850 & -1.376 & 8.59 \\
\hline ILE611 & VAL853 & -1.383 & 7.25 \\
\hline PHE612 & PHE852 & -1.660 & 10.05 \\
\hline PHE612 & VAL853 & -1.438 & 6.78 \\
\hline PHE612 & ASN856 & -0.857 & 8.95 \\
\hline THR614 & PRO858 & -0.434 & 7.49 \\
\hline ARG624 & PHE852 & -0.910 & 8.27 \\
\hline ASN627 & ILE849 & -0.741 & 8.46 \\
\hline LEU628 & ILE849 & -1.610 & 7.16 \\
\hline TYR631 & LEU845 & -1.296 & 8.62 \\
\hline ASP638 & ALA841 & -0.389 & 7.94 \\
\hline MET639 & ASN843 & -0.675 & 10.49 \\
\hline GLU655 & ASN843 & -0.345 & 8.15 \\
\hline LYS656 & ASN843 & -0.277 & 5.15 \\
\hline LYS659 & ILE844 & -0.688 & 6.53 \\
\hline ILE660 & LEU845 & -1.610 & 7.31 \\
\hline ILE660 & MET850 & -1.376 & 8.69 \\
\hline GLU663 & MET850 & -0.661 & 7.98 \\
\hline LEU664 & MET850 & -1.466 & 6.84 \\
\hline LEU664 & VAL853 & -1.482 & 8.27 \\
\hline LEU664 & LEU854 & -1.685 & 6.80 \\
\hline LYS667 & LEU854 & -0.771 & 6.92 \\
\hline ARG668 & LEU854 & -0.921 & 6.55 \\
\hline ARG671 & ASP851 & -0.524 & 10.36 \\
\hline ARG671 & LEU854 & -0.921 & 7.42 \\
\hline ARG671 & LYS855 & -0.135 & 7.56 \\
\hline Total & & -27.24 & \\
\hline
\end{tabular}




\section{SI References}

(1) Karanicolas, J.; Brooks, C. L., III. The Origins of Asymmetry in the Folding Transition States of Protein L and Protein G. Protein Sci. 2002, 11 (10), 2351-2361.

(2) Hills, R. D.; Brooks, C. L., III. Insights from Coarse-Grained Gō Models for Protein Folding and Dynamics. Int. J. Mol. Sci. 2009, 10 (3), 889-905.

(3) Miyazawa, S.; Jernigan, R. L. Residue-Residue Potentials with a Favorable Contact Pair Term and an Unfavorable High Packing Density Term, for Simulation and Threading. $J$. Mol. Biol. 1996, $256(3), 623-644$.

(4) Borgia, A.; Borgia, M. B.; Bugge, K.; Kissling, V. M.; Heidarsson, P. O.; Fernandes, C. B.; Sottini, A.; Soranno, A.; Buholzer, K. J.; Nettels, D.; et al. Extreme Disorder in an Ultrahigh-Affinity Protein Complex. Nature 2018, 555 (7694), 61-66.

(5) Ganguly, D.; Zhang, W.; Chen, J. Electrostatically Accelerated Encounter and Folding for Facile Recognition of Intrinsically Disordered Proteins. PLoS Comput. Biol. 2013, 9 (11), e1003363.

(6) Ganguly, D.; Chen, J. Topology-Based Modeling of Intrinsically Disordered Proteins : Balancing Intrinsic Structural Propensities and Intermolecular Interactions. Proteins Struct. Funct. Bioinforma. 2011, 79 (4), 1-5.

(7) Law, S. M.; Gagnon, J. K.; Mapp, A. K.; Brooks, C. L., III. Prepaying the Entropic Cost for Allosteric Regulation in KIX. Proc. Natl. Acad. Sci. 2014, 111 (33), 12067-12072.

(8) Brüschweiler, S.; Konrat, R.; Tollinger, M. Allosteric Communication in the KIX Domain Proceeds through Dynamic Repacking of the Hydrophobic Core. ACS Chem. Biol. 2013, 8 (7), 1600-1610.

(9) Liu, P.; Kim, B.; Friesner, R. A.; Berne, B. J. Replica Exchange with Solute Tempering: A 
Method for Sampling Biological Systems in Explicit Water; 2005; Vol. 102.

(10) Eastman, P.; Swails, J.; Chodera, J. D.; McGibbon, R. T.; Zhao, Y.; Beauchamp, K. A.; Wang, L. P.; Simmonett, A. C.; Harrigan, M. P.; Stern, C. D.; et al. OpenMM 7: Rapid Development of High Performance Algorithms for Molecular Dynamics. PLoS Comput. Biol. 2017, 13 (7), e1005659.

(11) Bussi, G. Hamiltonian Replica Exchange in GROMACS: A Flexible Implementation. Mol. Phys. 2014, 112 (3-4), 379-384.

(12) Goto, N. K.; Zor, T.; Martinez-Yamout, M.; Dyson, H. J.; Wright, P. E. Cooperativity in Transcription Factor Binding to the Coactivator CREB-Binding Protein (CBP): The Mixed Lineage Leukemia Protein (MLL) Activation Domain Binds to an Allosteric Site on the KIX Domain. J. Biol. Chem. 2002, 277 (45), 43168-43174. 